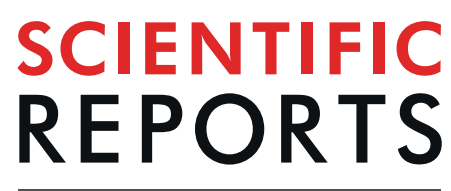

natureresearch

Received: 15 February 2019

Accepted: 6 September 2019

Published online: 20 September 2019

\section{Disrupted brain structural connectivity in Pediatric Bipolar Disorder with psychosis}

Henrique M. Fernandes ${ }^{1,2,3}$, Joana Cabral $\mathbb{D}^{1,2,4}$, Tim J. van Hartevelt ${ }^{1,2}$, Louis-David Lord ${ }^{2}$, Carsten Gleesborg ${ }^{3,5}$, Arne Møller ${ }^{3}$, Gustavo Deco, ${ }^{6,7}$, Peter C. Whybrow ${ }^{8}$, Predrag Petrovic ${ }^{9}$, Anthony C. James ${ }^{2,10}$ \& Morten L. Kringelbach $\mathbb{D}^{1,2,3,4}$

Bipolar disorder (BD) has been linked to disrupted structural and functional connectivity between prefrontal networks and limbic brain regions. Studies of patients with pediatric bipolar disorder (PBD) can help elucidate the developmental origins of altered structural connectivity underlying BD and provide novel insights into the aetiology of BD. Here we compare the network properties of wholebrain structural connectomes of euthymic PBD patients with psychosis, a variant of PBD, and matched healthy controls. Our results show widespread changes in the structural connectivity of PBD patients with psychosis in both cortical and subcortical networks, notably affecting the orbitofrontal cortex, frontal gyrus, amygdala, hippocampus and basal ganglia. Graph theoretical analysis revealed that PBD connectomes have fewer hubs, weaker rich club organization, different modular fingerprint and inter-modular communication, compared to healthy participants. The relationship between network features and neurocognitive and psychotic scores was also assessed, revealing trends of association between patients' $I Q$ and affective psychotic symptoms with the local efficiency of the orbitofrontal cortex. Our findings reveal that PBD with psychosis is associated with significant widespread changes in structural network topology, thus strengthening the hypothesis of a reduced capacity for integrative processing of information across brain regions. Localised network changes involve core regions for emotional processing and regulation, as well as memory and executive function, some of which show trends of association with neurocognitive faculties and symptoms. Together, our findings provide the first comprehensive characterisation of the alterations in local and global structural brain connectivity and network topology, which may contribute to the deficits in cognition and emotion processing and regulation found in PBD.

Bipolar disorder (BD) is a psychiatric illness characterized by episodes of mania or hypomania and depression interleaved with euthymic periods. It has been suggested that prefrontal control over emotional networks is deficient in $\mathrm{BD}$, inducing abnormally heightened emotional and reward processing in patients ${ }^{1-4}$.

Functional neuroimaging studies of euthymic bipolar patients have shown both reduced activity in the prefrontal cortex (PFC), especially in the ventrolateral and ventromedial PFC, and increased activity in emotional processing areas such as the ventral striatum ${ }^{5}$, the amygdala ${ }^{6}$ and the insula ${ }^{7,8}$ whilst performing a range of emotional processing tasks. Similar activity patterns in PFC were also observed in manic ${ }^{3}$ and depressed ${ }^{9,10}$ episodes. Moreover, functional connectivity studies have suggested a decreased coupling between frontal and limbic areas ${ }^{10}$, which might reflect abnormalities in the way these regions jointly process information in BD. 
In contrast, structural neuroimaging studies are not dependent on the choice of an experimental task. The need for higher statistical power and convergence of the findings has motivated the production of several large voxel-based meta-analyses ${ }^{11-14}$, as well as large single-centre studies ${ }^{15}$. These structural studies have found decreased brain volume in $\mathrm{BD}$ in a specific set of brain regions including medial $\mathrm{PFC}$, anterior cingulate cortex and ventrolateral PFC as well as the insula.

In addition to volumetric analyses, studies of structural connectivity (SC) in BD, which serves as the structural substrate over which the repertoire of functional networks can unfold, can provide highly valuable insights into the neural mechanisms underlying this disorder. Diffusion imaging studies have found altered connectivity between hemispheres ${ }^{16,17}$, as well as posterior cingulum ${ }^{17}$, prefrontal and subcortical regions in $\mathrm{BD}$, which may result in abnormal communication between these regions and disrupted modulation of emotion processing, onset of mania and the development of $\mathrm{BD}^{2,18-20}$.

In summary, there is emerging evidence linking $\mathrm{BD}$ to altered functional and structural connectivity between prefrontal regions and emotion processing regions such as the insula and the amygdala. BD most often starts in childhood or adolescence with sub-clinical or clinical manifestations of the disorder, suggesting an important developmental component ${ }^{21}$. Pediatric bipolar disorder (PBD) is a cyclic mood disorder in children and adolescents, which can be persistent and severe, and has an estimated prevalence of around $1 \%{ }^{22}$. Few studies have explored the connectivity patterns in the neurodevelopmental phase of adolescence, when active brain reorganisation is occurring. Early-onset bipolar disorder with psychosis, a variant of PBD, represents a useful, possibly, intermediate state to examine. Frontal connectivity alterations are common to both schizophrenia and bipolar disorder, with prominent fronto-temporal deficits identified in young patients with bipolar disorder ${ }^{23}$, as well as in schizophrenia, and inter-hemispheric and limbic alterations reported in bipolar disorder ${ }^{23,24}$.

Although abnormal functional connectivity is proposed to be a main feature in BD, few studies have focused on whether the underlying structural connectivity is altered in BD using state-of-the-art whole-brain network analysis ${ }^{23,25-30}$. As such, complementing the existing knowledge of anatomical and functional deficits in PBD with a comprehensive mapping of altered structural connectivity will inform how brain structure-function coupling is affected, and thus allow for an improved assessment of patients' cognition ${ }^{28}$. Furthermore, this may help to minimise potentially confounding secondary manifestations of $\mathrm{BD}$, which is key to elucidate which neural mechanisms are inducing and maintaining manic and depressed states in BD.

Here we hypothesize that PBD is characterised by reduced ability to integrate information across brain regions, driven by widespread changes in structural network topology, and predominantly affecting key areas for emotion processing and regulation. Furthermore, changes in local network properties can predict neurocognitive scores and psychotic symptoms. To test this, we constructed the structural connectomes of a group of adolescents with PBD and psychosis and of a group of closely matched healthy controls. We used advanced structural connectomics analysis to characterise the topological differences in structural connectivity driving the cognitive and affective psychotic symptoms found in PBD with psychosis.

\section{Methods}

Participants. We analysed data from 15 patients with PBD with psychosis from the Oxford regional unit and surrounding units, and 15 euthymic age- and gender-matched healthy controls (HC) (Table 1). The patients were diagnosed according to the DSM-IV-TR criteria using the Kiddie Schedule for Affective Disorders and Schizophrenia (K-SADS-PL), and were administered the Positive and Negative Syndrome Scale (PANSS). The healthy subjects were recruited from the community through their general practitioners and interviewed using the K-SADS-PL to rule out any history of emotional, behavioural, or medical problems. Handedness was assessed with the Edinburgh Handedness Questionnaire. Intellectual ability, given by the verbal Intelligence Quotient (IQ), the performance IQ and the Full Scale Intelligence Quotient (FSIQ), was assessed using the Wechsler Abbreviated Scale of Intelligence (WASI). All subjects were clinically reviewed after the initial diagnostic screening for a period of at least six months (mean \pm std; $10 \pm 1.5$ months); no subject changed diagnostic status. James and colleagues previously reported a comprehensive description of the cohort and clinical assessment strategy used in this study ${ }^{2}$. This study was undertaken in accordance with the guidance of the Oxford Psychiatric Research Ethics Committee (OPREC). All clinical and neuroimaging protocols included in this study were approved by the OPREC. Informed consent was obtained from all participants. Consent was obtained from the parents/legal guardians in cases where the participants were under the age of 18 .

Image acquisition. All 30 participants underwent the acquisition of whole-brain T1-weighted and diffusion-weighted images using a 1.5T Sonata magnetic resonance imager (Siemens, Erlangen, Germany) with a standard quadrature head coil and maximum $40 \mathrm{mT} / \mathrm{m}$ gradient capability. The 3D T1-weighted FLASH sequence was performed with the following parameters: coronal orientation; $256 \times 256$ reconstructed matrix; 208 slices; $1 \times 1 \mathrm{~mm}^{2}$ in-plane resolution; slice thickness of $1 \mathrm{~mm}$; echo time (TE) of $5.6 \mathrm{~ms}$; repetition time (TR) of $12 \mathrm{~ms}$; flip-angle $(\alpha)$ of $19^{\circ}$. The diffusion-weighted sequences were obtained using echo-planar imaging (SE-EPI), and its scanning parameters were: TE of $89 \mathrm{~ms}$; TR of $8500 \mathrm{~ms}$; 60 axial slices; bandwidth $=1860 \mathrm{~Hz} / \mathrm{vx}$; voxel size of $\left.2.5 \times 2.5 \times 2.5 \mathrm{~mm}^{3}\right) ; 60$ isotropically distributed orientations for the diffusion-sensitising gradients at a b-value of $1000 \mathrm{~s} / \mathrm{mm}^{2}$ and five b0 images. To increase signal-to-noise ratio, scanning was repeated three times and all scans were merged.

Network construction. The construction of the brain structural network for each experimental group consisted of a two-step process. First, the nodes of the network were defined using a brain parcellation. Secondly, the connections between nodes (i.e. edges) were estimated using probabilistic tractography. In the following we outline the details involved in each step. 


\begin{tabular}{|c|c|c|c|c|c|}
\hline \multirow[b]{2}{*}{ Characteristic } & \multicolumn{2}{|c|}{ Normal Controls } & \multicolumn{2}{|c|}{ Patients with PBD } & \multirow[b]{2}{*}{ p-value } \\
\hline & mean & SD & mean & SD & \\
\hline Gender, M/F & $8 / 7$ & - & $8 / 7$ & - & 1 \\
\hline Handedness, R/L & $13 / 2$ & & $13 / 2$ & & 1 \\
\hline Age at onset of symptoms, years & 15.70 & 1.26 & 15.04 & 2.04 & 0.30 \\
\hline Disease duration, years & & & 14.00 & 2.00 & \\
\hline Verbal IQ & 103.60 & 19.66 & 94.80 & 17.03 & 0.20 \\
\hline Performance IQ & 106.53 & 12.98 & 99.13 & 15.01 & 0.16 \\
\hline Full Scale IQ & 105.47 & 16.37 & 96.33 & 16.04 & 0.13 \\
\hline Coding & - & - & 81.33 & 19.77 & - \\
\hline PANSS: positive scores & - & - & 19.27 & 3.92 & - \\
\hline PANNS: negative scores & - & - & 10.13 & 2.72 & - \\
\hline Beck Depression Inventory & & & 6.60 & 1.40 & \\
\hline Young Mania Rating Scale & & & 1.40 & 0.80 & \\
\hline Comorbidity (ADHD), n & & & $5 / 15$ & & \\
\hline \multicolumn{6}{|l|}{ Medication, $\mathrm{n}$} \\
\hline Olanzapine & & & 7 & & \\
\hline Quetiapine & & & 3 & & \\
\hline Risperidone & & & 1 & & \\
\hline Fluoxetine & & & 1 & & \\
\hline Sodium valproate & & & 2 & & \\
\hline Lithium & & & 3 & & \\
\hline
\end{tabular}

Table 1. Demographic and clinical characteristics of participants. PANSS $=$ Positive and Negative Syndrome Scale; $\mathrm{ADHD}=$ attention-deficit hyperactivity disorder.

Brain parcellation. For each subject, the brain was parcellated in native DTI space into 90 different cortical and subcortical regions using the Automated Anatomical labelling (AAL) template ${ }^{31}$, where each region represents a node in the brain network.

Flirt (FMRIB, Oxford) ${ }^{32}$ was used to linearly co-register the standard ICBM152 in MNI space ${ }^{33}$ to each native T1-weighted structural image of each subject, using an affine registration (12 DOF) combined with a nearest-neighbour interpolation method. The resulting transformation was further applied to warp the Automated Anatomical Labeling (AAL). The MRI-DTI scan was then converted to MRI-T1 space, using a rigid-body transformation (6 DOF) and the resulting transformation matrix inverted. The MNI to MRI-T1 and MRI-T1 to MRI-DTI transformation matrices were subsequently concatenated, allowing a direct co-registration of the AAL template in MNI space to the diffusion MRI native space. This last transformation was performed using a nearest-neighbour interpolation method to ensure that discrete labelling values were preserved (see Supplementary Fig. 2 for an example of the outputs described above, for a single subject).

Brain structural network. We used the FDT toolbox in FSL (version 5.0, http://www.fmrib.ox.ac.uk/fsl/, FMRIB, Oxford) to carry out the multiple processing stages of the diffusion MRI data. The initial pre-processing involved correction of head motion and eddy current gradient induced image distortions. Subsequently, we modelled for crossing fibres within each voxel of the brain using a Markov Chain Monte Carlo sampling algorithm to build up distributions on diffusion parameters and estimate the local probability distribution of fibre direction at each voxel of the brain ${ }^{34}$. For this step, we used an automatic estimation of two fibre directions within each voxel, in order to improve the tracking sensitivity of non-dominant fibre populations in the human brain ${ }^{35}$.

Probability of connectivity was estimated using probabilistic tractography at the voxel level, with a sampling number of streamline fibres per voxel set at 5000. Brain boundaries were defined based on a binary brain previously registered in a skull-extracted version of the subject's native brain. The connectivity from a seed voxel $i$ to another voxel $j$ was defined by the proportion of streamlines leaving voxel $i$ and reaching voxel $j^{35}$. This was then extended from the voxel to the region level, i.e. in a brain region consisting of $n$ voxels, $5000 * n$ fibres were sampled. The connectivity probability $P_{i j}$ from region $i$ to region $j$ is then calculated as the number of sampled fibres in region $i$ connecting the two regions, divided by $5000^{*} n$, where $n$ is the number of voxels in region $i$. For each brain region, the connectivity probability to each of the remaining $89 \mathrm{AAL}$ regions was calculated. The regional connectivity probability was computed using in-house Perl scripts and further normalised by each region's volume, expressed in number of voxels. It should be noted that, given the dependence of tractography on the seeding location, the probability from $i$ to $j$ is not necessarily the same to that from $j$ to $i$. However, these two probabilities are highly correlated across the brain for all participants. We therefore defined the undirectional connectivity probability $P_{i j}$ between regions $i$ and $j$ by averaging these two probabilities. We considered this as a measure of the structural connectivity between each pair of areas, with $C_{i j}=C_{j i}$.

For each participant, a $90 \times 90$ symmetric weighted matrix $C$ was constructed, representing the brain's structural network. To remove spurious connections (i.e. control for false positives resultant from probabilistic tractography $)^{36}$, we applied a threshold to each individual SC at $1 \%$ of their strongest connection (i.e. removed the $1 \%$ bottom-ranked links in terms of connection strength). The outcome of this strategy was fully convergent 
with a consistency-based network thresholding technique ${ }^{37}$. Given the potential systemic effects of head motion on the presentations of the connectome ${ }^{38,39}$, the levels of head-motion in a diffusion scan (using FSL tools) were assessed for both cohorts. To evaluate the potential impact of group differences in data quality on our estimations of SC, we analysed the levels of intra- and inter-group consistency in the resulting signatures of individual SC (Supplementary Information; Supplementary Figs 3 and 4).

Graph theoretical analysis. The structural brain networks, represented as a $90 \times 90$ connectivity matrix, can be analysed as graphs. Using the Brain Connectivity Toolbox ${ }^{40}$, the brain networks were characterized using measures from graph theory. A rescaled version of the structural networks, where individual networks are normalised by its maximum connectivity value, was used. The following standard local and global graph metrics were considered: Connection Density, Node Degree, Local Efficiency, Global Efficiency, Characteristic Path length, Clustering Coefficient and Small-World index. Definitions and equations for these measures can be found in Supplementary Information. Additionally, the following topological features were assessed for both populations.

Modularity. Modularity quantifies the degree to which the network may be subdivided into non-overlapping communities. Therefore, the optimal community structure is represented as a subdivision of the network into well-defined groups of nodes so that it maximizes the number of within-group edges and minimizes the number of between-group edges. The calculation of the modularity coefficient was determined using the Louvain algorithm ${ }^{41}$. To circumvent the probabilistic nature of this algorithm, the optimal partition was selected from an iterative set of 1000 partitions, based on modularity maximisation statistic, i.e. the degree to which the network may be subdivided into clearly delineated groups.

Hubs. In this study, network hubs are defined as the most efficient nodes in a network. The local efficiency of a node $\left(E_{\text {nodal }}\right)$ measures its connectivity to all other nodes of the network. Hence, highly connected nodes or "hubs" will have high regional efficiency ${ }^{42}$. For each node $i$, if the normalised (divided by the mean $E_{\text {nodal }}$ of all nodes) $E_{\text {nodal }}(i)$ is larger than the normalised mean $E_{\text {nodal }}$ of all nodes in a network plus one standard deviation (SD), the node is considered a hub region ${ }^{43}$.

In addition to the hub classification based on $E_{\text {nodal }}$, a set of hubs were classified by analysis of the distribution of connections within and between modules, allowing the categorization of hub regions into provincial and connector hubs, as described below.

Provincial hubs were defined as the nodes with a high within-module degree centrality (within module z-score greater than the mean plus SD of all nodes), indicating its central role in intra-modular communication, and low participation coefficient $(\mathrm{PC})(\mathrm{PC} \leq 0.3)$. Participation coefficient or proportion of cross-module connectivity profile compares the number of links of a given node to other nodes in different communities, to the total number of links to other nodes in the same community. This measure is defined as:

$$
P C(i)=1-\sum_{c=1}^{N_{C}}\left(\frac{k_{C_{i}}}{k_{i}}\right)^{2},
$$

Connector hubs were defined as regions with a high value of within-module degree centrality and participation coefficient $(\mathrm{PC}>0.3)$, indicating a high proportion of cross-module connectivity, and thus a central role in the inter-modular communication. Upon identifying connector-hubs, we further characterized their connectivity to the rest of the network.

Rich club. By definition, a set of brain areas in the network shows a rich club structure if its level of interconnectivity exceeds the level of connectivity expected on basis of chance alone ${ }^{40}$. In particular, the weighted rich club coefficient $\Phi(k)$ is computed as the ratio between the weights of connections present within the subnetwork $S$, composed of regions with a degree $>\mathrm{k}$, and the total sum of weights present within an equally sized subset of the top ranking connection-weights in the network. The normalized rich club coefficient $\Phi_{\text {norm }}(k)$ is computed by dividing $\Phi(k)$ by $\Phi_{\text {random }}(k)$, with $\Phi_{\text {random }}(k)$ calculated as the average rich club coefficient for each $k$ of a set of 1000 randomized graphs (preserving the degree distribution $)^{40}$. Therefore, a structural brain network can be described as having a rich club organization if, for a given degree interval, the normalized rich club coefficient is greater than 1.

The integrative nature of rich club members by the linking of different communities was also assessed. For this, we identified "rich-connector-hub networks". These were constructed by examining the connectivity profile of nodes that simultaneously qualified as rich-club members and connector hubs (as defined above), to the remaining connector hubs in the network.

Between-group differences in structural connectivity strength. Between-group statistical comparison of the raw structural connectomes for PBD patients and controls was performed using network-based statistics (NBS), a non-parametric statistical method that allows the identification of significantly altered sub-networks, while controlling for the family-wise error rate (FWER $)^{44}$. In an initial step, NBS performs between-group comparisons on a connection-by-connection basis using t-statistics and a primary threshold is set to define potential supra-threshold connected candidates for which the null hypothesis can be rejected in the subsequent level of the NBS analysis. Importantly, FWER is guaranteed in NBS irrespective of the primary threshold choice, which affects only the sensitivity of the method. The primary $\mathrm{t}$-threshold for this specific dataset was set to $t=2.1$, which was the maximal $t$-threshold where a connected component was detected (see Supplementary Fig. 1). We note that this same $t$-threshold was recently found to reveal abnormal brain networks in schizophrenia ${ }^{45}$. Group 
effects in structural connectivity detected with NBS are represented by significantly connected components with FWER-corrected $p<0.05$.

Between-group statistical differences in graph measures. Between-group statistical comparison was performed using M-W U test, K-S test and t-test.

Fingerprints of modular connectivity - within- and between-group analysis. In order to investigate potential alterations in modular connectivity of PBD with psychosis, we analysed the network fingerprints of inter-modular (global and connector-hub-driven) and intra-modular structural connectivity, for each group. Modular connectivity strength was quantified as the total number of connections (degree) of all nodes forming a module. In order to allow for group comparison, a single community structure was used as the reference scheme to quantify modular connectivity for both populations. The criteria for selecting the reference scheme was based on which population revealed a higher mean score of community-structure goodness-of-fit, i.e. how well the estimated group community structure represents each subject's individual community structure. Maps of 'between-module connectivity', 'within-module connectivity' and 'connector-hub modular connectivity' were then produced for each of the two populations.

The topological characterisation of each module was done by computing the mean degree, participation coefficient, betweeness-centrality and within-module degree centrality of all nodes in a module's network. Group differences in modular network topology were investigated using non-parametric statistical testing (permutation-based unpaired t-tests). Correction for multiple comparisons was performed using FDR-correction.

Association between network metrics and neurocognitive scores and symptoms. The neurocognitive scores (coding and FSIQ) assessed for the HC and PBD groups, as well as the positive and negative affective psychotic symptoms scores (PANSS) assessed in the PBD patients, were correlated with local and global network properties. Partial correlation analysis was performed, using age and gender as confounding variables. Similarly, the correlation between clinical scores and the subset of network nodes revealing significant group differences in nodal efficiency were also assessed using age and gender as confounding variables.

\section{Results}

Demographics. No significant differences were found for age $(p=0.30)$, gender $(p=1)$, verbal IQ $(p=0.20)$, performance IQ $(p=0.16)$ and FSIQ $(p=0.13)$ scores between patients with Pediatric Bipolar Disorder (PBD) with psychosis and healthy controls (HC) (Table 1$)$.

Data quality and group consistency. Both cohorts showed high levels of intra-group consistency in the estimation of whole-brain SC, as well as the SC of a subnetwork comprising regions with increased susceptibility to signal dropout (OFC and temporal pole) in diffusion sequences (Supplementary Figs 3 and 4). Despite a trend for higher relative head motion found for the patient group (HC: $1.39 \mathrm{~mm} \pm 0.27 \mathrm{vs}$. PBD: $1.84 \mathrm{~mm} \pm 0.83$, $\mathrm{t}$-test $p=0.06$ ), the strong group consistency levels suggest an efficient minimisation of potential artefact sources (such as head displacement, eddy-currents), thus strengthening the reliability of our findings.

Network connectivity changes. Compared to controls, patients with PBD showed significantly altered structural connectivity $(p=0.015)$ in a brain sub-network, or connected component, involving 71 structural links ( $1,8 \%$ of all possible connections; $\sim 5,5 \%$ of all existing connections) (Fig. 1). These 71 links showed 39 decreases and 32 increases in connectivity strength in the PBD with psychosis group (see Supplementary Table 1 for details). The network is predominantly distributed in right frontal inferior and temporal brain areas (22\% more connections on the right hemisphere).

Graph theoretical analysis: local properties. Nodal efficiency-network hubs. For each group, regions showing a high normalized nodal efficiency were identified as network hubs. Between-group differences found in the network hub configuration are suggestive of local asymmetries in efficiency of communication (Fig. 2).

In the HC group, 13 hub areas were identified (Fig. 2c; Supplementary Table 2). The hub configuration for the $\mathrm{PBD}$ with psychosis group revealed the loss of five hubs and inclusion of one new hub when compared to the HC group. Of particular interest are the exclusivity of the medial orbitofrontal and inferior temporal (bilateral) hubs to healthy controls.

Graph theoretical analysis: global properties. No significant global topological differences were found between groups on any of the global graph measures assessed: global efficiency, modularity, clustering coefficient, characteristic path length and small-worldness (see Supplementary Table 5).

Modularity and hub classification. For each group, the whole-brain optimal community structure was decomposed into seven modules as shown in Fig. 3A. The HC and PBD with psychosis groups revealed differences in their modular arrangement, with the most important changes found in the right lateral and the medial posterior regions of the brain.

Rich club. Rich club organisation was found in the structural networks of both HC and PBD with psychosis patients. The group-weighted and normalised rich club coefficient curves of both groups, shows that the maximal $\Phi_{\text {norm }}(k)$ is reached at $k=35$ for both groups. However, as illustrated in Fig. 4A, the peak amplitude is clearly decreased in the patient group, which is indicative of a reduced rich club organisation for this group. This reveals lower level of connectivity between the most densely connected regions in the brain, when compared with the 
A

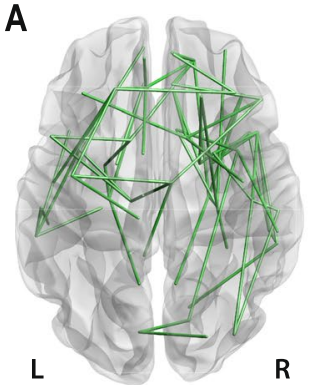

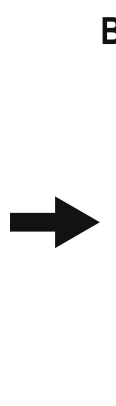

(

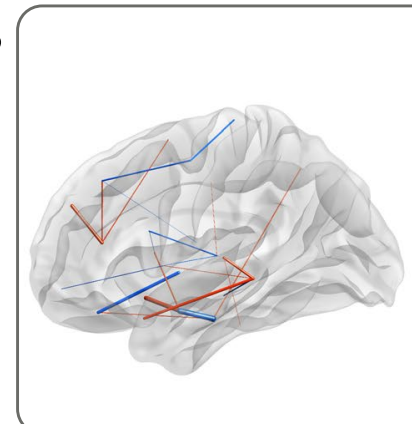

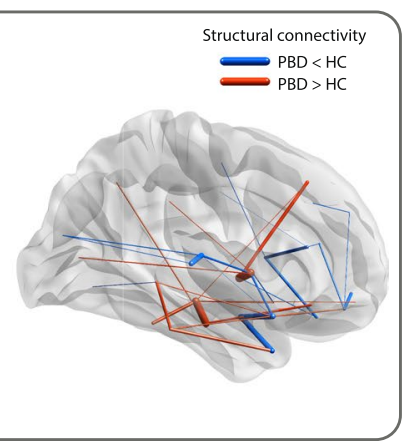

Figure 1. Significant changes in the structural connectivity of patients with pediatric bipolar disorder (PBD) with psychosis, compared to healthy controls (HC). Connected component of significant differences in structural connectivity strength between patients with $\mathrm{PBD}$ and $\mathrm{HC}$, represented with edges connecting a pair of regions. (A) Binarised version of the connected network of significantly altered structural connectivity in PBD. (B) Weighted version of (A), where the edge thickness represents the amplitude of the differences in structural connectivity. Decreases and increases in connectivity strength between regions in the PBD group are represented in blue and red respectively. PBD primarily differs from HC in inferior temporal networks (e.g. left and right temporal pole to left and right amygdala, left amygdala to left parahippocampal region) and inferior frontal networks (e.g. right orbitofrontal cortex) and subcortical to inferior frontal regions (e.g. right caudate to orbitofrontal cortex and left pallidum to left orbitofrontal cortex).

HC group. This effect appears clear and reflects the interval of largest difference between the two curves. However, given that it only extends for a limited range of $k$ values $(34 \leq k<37)$, it is not sufficiently large to reject the null hypothesis of no difference between means. Additionally, rich club membership between groups differed only in two right hemisphere nodes, suggesting that the insular cortices and the hippocampus have a rich club profile exclusive to the patient group (Fig. 4B).

As described in the Methods section Rich Club, we also examined the connectivity profile of nodes that simultaneously qualified as rich-club members and connector hubs to the other connector hubs in the network (Fig. 4C). These networks revealed a relatively symmetric connectivity pattern and central spatial distribution in the HC group, involving regions of the right posterior cingulum and amygdala, left precuneus and left and right hippocampus and putamen (Fig. 4C (bottom)). This strong 'core-effect', supported by a structural network of rich-connector-hubs linking functionally different communities, reflects a tendency toward efficient integration of information across the brain, in the HC group. In contrast, the patient group exhibited a comparatively asymmetric profile of 'rich-connector-hub' connectivity (Fig. 4C (top)). This tendency toward spatially unbalanced modular integration was characterised by a deficit of two right hemisphere rich-connector-hubs (loss of the hippocampus, amygdala and putamen; gain of the superior occipital gyrus), when compared to the HC group, resulting in a single right hemisphere module (posterior) being comprised in this network, as opposed to two right-hemisphere modules in the HC group.

Modular connectivity. Our findings suggest that there are relevant alterations in the fingerprints of structural inter-modular connectivity in PBD with psychosis (Fig. 5). Specifically, in the patient group, the connectivity of module 4 (right hemisphere; thalamus, insula, basal ganglia, anterior cingulate, precentral gyrus and regions covering most of the frontal cortex) is increased to module 1 (right hemisphere; amygdala, hippocampus, parahippocampus, fusiform gyrus, and other regions of the occipital and temporal cortices) and decreased to module 3 (left hemisphere; thalamus, basal ganglia, anterior cingulate and a large extension of the frontal cortex). Overall, module 1 has increased inter-modular connectivity but decreased connector-hub driven inter-modular connectivity, which suggests that PBD with psychosis is associated to an increased degree of sparsity in inter-modular linkage (i.e. more connections, but a less prominent role of its connector-hubs in mediating inter-modular connectivity). Additionally, despite the absence of connector-hubs in module 4 for the patient group, its mean inter-modular connectivity strength is the second largest across all modules and remains approximately the same as in HC (with two connector-hubs). This suggests that more nodes (with no 'hub' or 'connector' profile - lower degree and lower participation coefficient) are mediating communication between module 4 and all other modules in PBD with psychosis, which may be indicative of a decreased efficiency/selectivity in communication processes between a network including left hemisphere limbic, insular and thalamic areas, and the rest of the brain.

Significant group differences in modular network topology were found in degree (modules 2 and 4 ), within-module degree centrality (module 7) and participation coefficient (module 6) (the full results are reported in Supplementary Table 6). However, only the last significant finding (module 6 - participation coefficient) survived correction for multiple comparisons.

Network properties vs. neurocognitive and psychotic symptoms. We examined the potential association of neurocognitive scores (coding and FSIQ) and affective psychotic symptoms from the PANSS, namely excitement (P4EX; positive), grandiosity (P5GR; positive), blunted affect (N1BA; negative) and emotional 

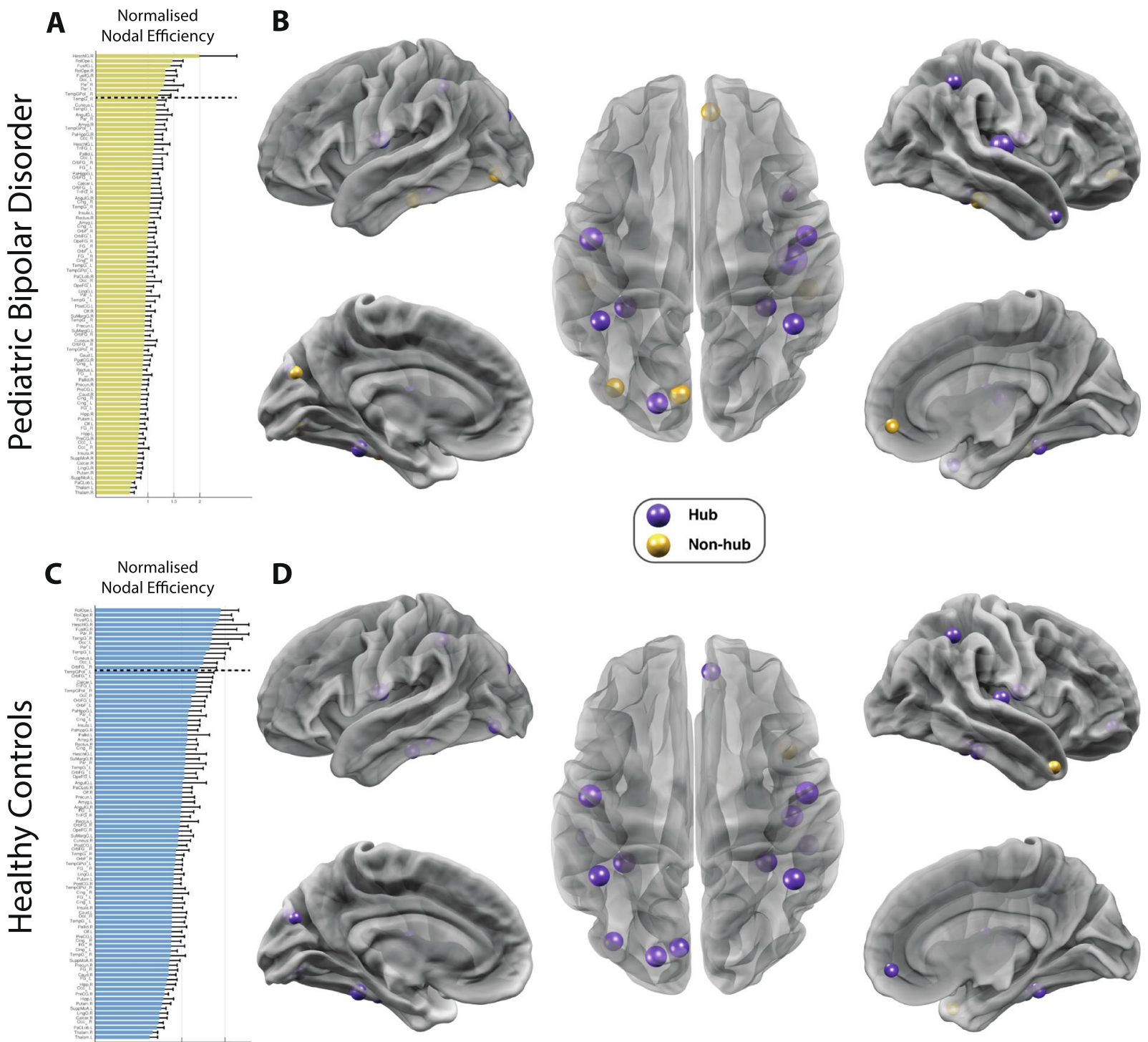

Figure 2. Altered landscape of network hubs in pediatric bipolar disorder (PBD) with psychosis. Differences in network hub regions in HC (top row) and PBD (bottom row) as measured by the normalised nodal efficiency for all 90 AAL brain regions. This shows a clear reorganisation in hub regions in PBD where e.g. the orbitofrontal cortex is no longer a hub region. (A) For the patient group, the figure shows the nodal efficiency sorted according to the mean in descending order. For each node, the $E_{\text {nodal }}$ is normalised by the mean of all nodes' $E_{\text {nodal }}$, and a node is identified as a hub region if its normalised $E_{\text {nodal }}$ is larger than the sum of the mean plus the SD of all network nodes' $E_{\text {nodal }}$ (B) Hub regions, represented as spheres positioned according to the centroid stereotaxic coordinates of the correspondent anatomical region, with node size proportional to their $E_{\text {nodal }}$, are mapped onto a 3D reconstructed brain surface. (C) The ranking of nodal efficiency, and (D) the identified hub regions for healthy participants. For each group, the existing hub nodes are represented in purple and non-hub nodes (hubs exclusive to the other group) in yellow.

withdrawal (N2EW; negative), with the significant group differences found in nodal efficiency (reported in Supplementary Table 3), as well as in global graph properties (Supplementary Table 4).

It is only for the orbitofrontal cortex that nodal efficiency was correlated with IQ and affective psychotic symptoms. Nodal efficiency of the left inferior orbitofrontal cortex was found to be positively correlated with the VIQ $(\mathrm{r}=0.74 ; p=0.04)$, PIQ $(\mathrm{r}=0.60 ; p=0.03)$ and FSIQ $(\mathrm{r}=0.77 ; p=0.02)$ scores, whereas emotional withdrawal (negative affective psychotic symptom) was positively correlated with the nodal efficiency of the left middle orbitofrontal cortex $(\mathrm{r}=0.57 ; p=0.04)$ (Fig. 6). These findings however did not survive correction for multiple comparisons.

None of the global network metrics assessed showed significant $(p<0.05$, uncorrected) correlation with the neurocognitive scores or psychotic symptoms (Supplementary Table 4). 
A

Connector-Hub

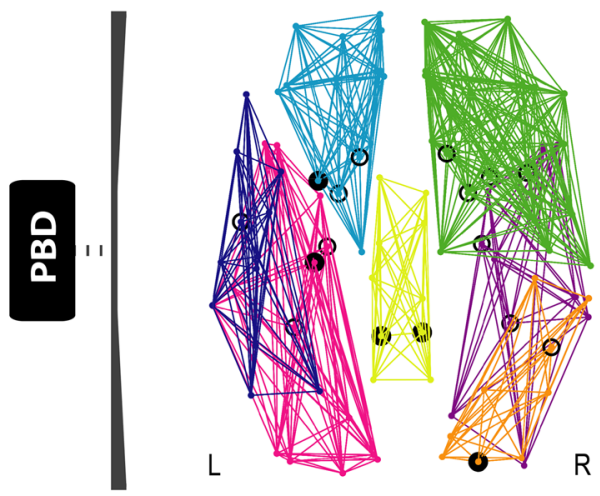

$\mathrm{R}$
B

Modules \& Hubs

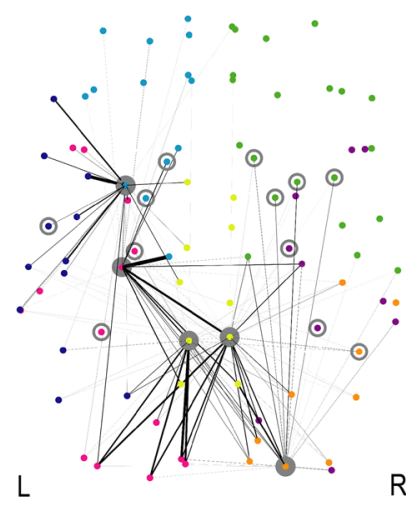

$\mathrm{R}$
C Connectivity Backbone

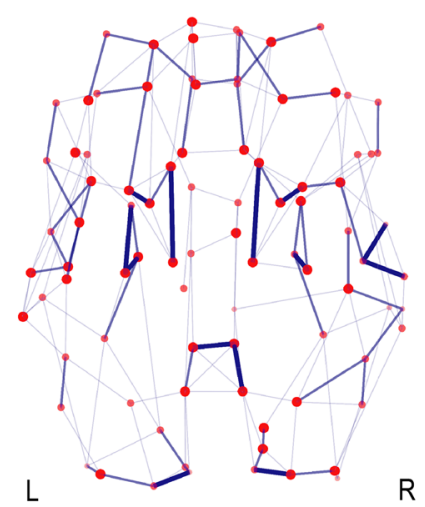

R
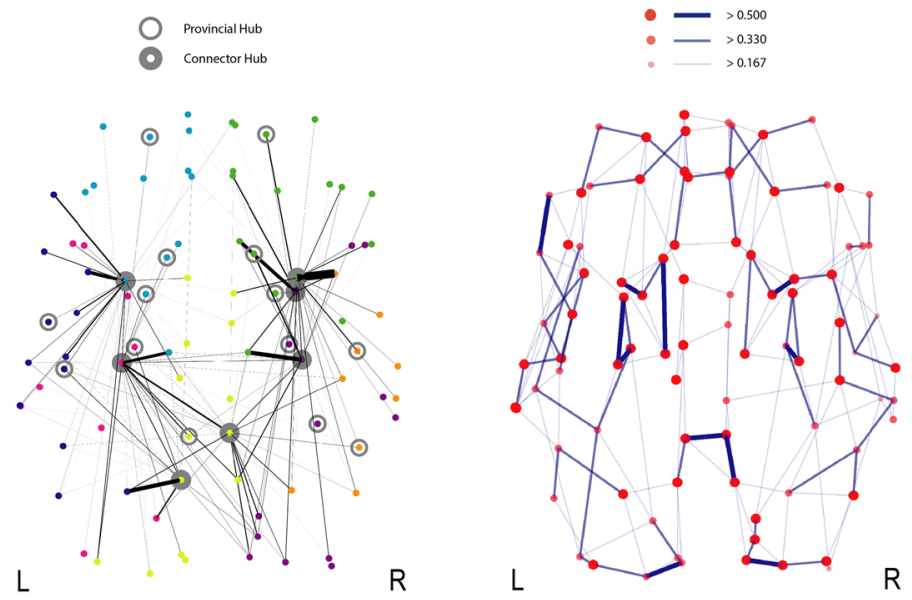

$\mathrm{R}$

Figure 3. Disrupted modularity, hubs and connector-hub connectivity in patients with pediatric bipolar disorder (PBD) with psychosis. The figure shows the changes in the optimal community structure, i.e. the seven modules and connector hubs shown on a dorsal view of the brain of patients with $\mathrm{PBD}$ (top row) and healthy controls (HC, bottom row), with node colours denoting membership to a module and with intra-module edges coloured accordingly. Connector hubs (marked as filled circles) are defined as regions with high withinmodule degree centrality and a participation coefficient $\mathrm{p}>0.3$, denoting a high proportion of cross-module connectivity. Provincial hubs (marked as unfilled circles) are defined as having high within-module degree centrality but participation coefficient $p \leq 0.3$. (A) Dorsal view of the optimal modularity partitioning found in PBD (top), reveals a different configuration compared to HC (bottom). (B) Significant local changes are found in the undirected structural connectivity profile for the connector hubs in PBD (top) compared to HC (bottom). Edges, coloured in black, correspond to the structural connectivity of each connector hub region to any other region in the brain, with thickness proportional to its connection strength. Colour of dots represents the belonging of a brain area to a particular community, consistent with (A). Albeit the number of both provincial and connector hubs is preserved between the two groups (Supplementary Table 2), variance in the distribution reflects the high impact on the regional dispersion and density of connector-hub-connectivity (CHC), i.e. network of undirected connections involving connector hubs. Whilst there is an almost symmetric distribution of provincial and connector hubs in the $\mathrm{HC}$ group, asymmetries found in the hub distribution in the PBD group led to a significantly decreased $\mathrm{CHC}$ density in the right anterior hemisphere and a shift of the CHC towards posterior areas of the brain, as shown in (B). Notably, the connection between the orbitofrontal provincial hub and the amygdala connector hub is missing in the patient group. (C) Dorsal view of the brain connectivity backbone for patients with PBD with psychosis (top), and HC (bottom). Nodes and edges are coded according to normalised degree and connection weight respectively. Diameter, thickness and transparency of nodes and edges are divided into three weight levels (see legend).

\section{Discussion}

We investigated the topological organisation of the structural connectomes in a cohort of adolescents with a diagnosis of PBD with psychosis. Network analysis using a combination of state-of-the-art methods revealed significant differences in structural connectivity between patients and healthy matched participants. Most differences were found in networks involved in emotional regulation, strengthening the hypothesis that BD could be linked to prefrontal top-down dysregulation of emotional processing. Specifically, we found widespread group differences in structural connectivity strength, and altered hub configuration and connectivity profile. Changes were also found in the rich-club organisation and inter-modular connectivity driven by 'rich-connector-hubs'. 
A

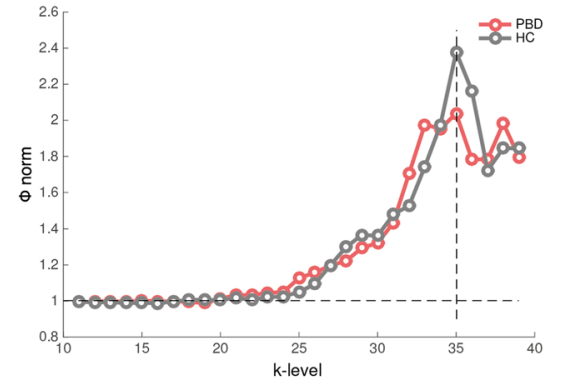

B

B

'Rich club' Network

PBD

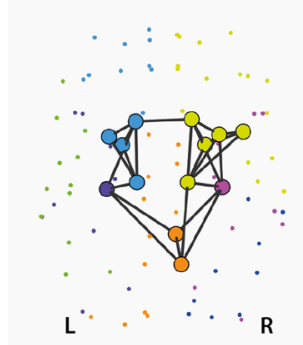

HC

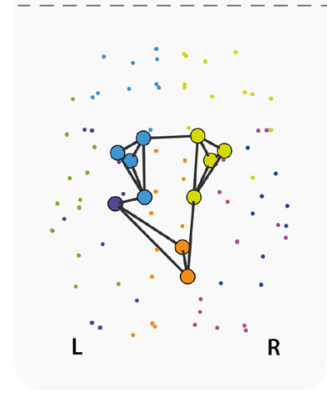

C 'Rich-Connector-Hub' Network

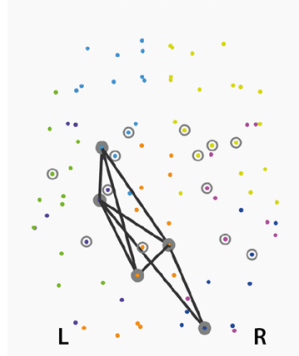

$\mathbf{L}$

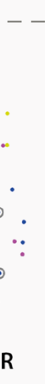

Figure 4. Altered rich club organisation and inter-modular integration in pediatric bipolar disorder (PBD). (A) The figure shows significant changes in the rich club organisation as demonstrated by the normalised weighted rich club coefficient as a function of $k$-level (degree), for the PBD (red curve) and healthy control (HC, grey curve) groups. (B) Rich-club members in HC (bottom) and PBD (top). Rich club nodes are represented by large filled circles with colours denoting the module to which they belong. Rich club member regions common to both groups included the left hippocampus, right posterior cingulate cortex and precuneus, and left and right caudate, putamen, pallidum and thalamus. Membership between groups differed only in two right hemisphere nodes, suggesting that the insular cortices and the hippocampus have a rich club profile exclusive to the PBD group. (C) Equally, the 'rich-connector-hub' network signature, i.e. the backbone of inter-modular connectivity driven by connector-hubs, differs between the HC (top) and PBD (bottom). Edges coloured in black represent the existing structural connectivity between connector hubs.

These findings reveal that key network features for balanced whole-brain integration of information are altered in PBD. In addition, our results suggest that the nodal efficiency of regions important for the regulation of emotional processing such as the orbitofrontal cortex show significant positive correlations with IQ score and emotional withdrawal in PBD patients with psychosis. Our results are in line with recent evidence of association between altered structural connectivity in a cortico-striatal reward circuit, particularly involving the OFC, and hypomania symptoms ${ }^{46}$. Overall, these findings of abnormal topological organization in PBD with psychosis shed new light on the potential neurobiological deficits underlying BD.

Alterations in structural connectivity were detected in the brain networks of PBD patients with psychosis using NBS at a relatively liberal primary threshold $(t=2.1$, FWER-corrected). This indicates that the effects are subtle but topologically extended (unlike effects present only at conservative thresholds, which are generally characterised by strong focal differences ${ }^{44}$ ). The alterations primarily differ from healthy controls in inferior temporal networks, inferior frontal networks and subcortical to inferior frontal regions (Fig. 1; Supplementary Table 1). The decreases in connectivity for PBD vs HC fit well with previously reported localised changes in white matter, where there is evidence for a loss of connectivity involving prefrontal and frontal regions through associative and commissural fibres ${ }^{47,48}$. Widespread decreases in fractional anisotropy (FA) have been found in major tracts, including (but not limited to) the orbitofrontal corte $\mathrm{x}^{20}$, superior frontal lobes ${ }^{16,48}$, and bilateral parietal and occipital corona radiate ${ }^{19}$. In contrast, the increases in connectivity in PBD vs $\mathrm{HC}$ are indicative of complex rewiring that leads to malfunctioning. It has indeed been suggested that white matter changes could be central to BD, and may represent an endophenotype ${ }^{49}$. Our findings of widespread connectivity changes in PBD with psychosis support this idea and add significant information on the specific structural connectivity networks and brain topological features that are affected.

Our structural connectivity findings are also consistent with the previously reported widespread changes in regional grey matter volumes in PBD. Specifically, neuroimaging studies have linked $\mathrm{PBD}^{50}$ to altered cortical grey matter volumes in several regions shown to have abnormal structural connectivity in the present study, including (but not limited to) the basal ganglia ${ }^{51}$, thalamus ${ }^{51}$ and temporal cortices ${ }^{51,52}$, cingulate ${ }^{50-53}$, dorsolateral prefrontal cortex (DLPFC) ${ }^{54,55}$, temporal lobe ${ }^{2,51,56}$, orbitofrontal cortex (OFC) $)^{2,51,54,55}$, and amygdala. The substantial overlap between previously reported grey matter changes and the structural connectivity changes demonstrated here, are suggestive of a mechanistic link between the volume of cortical and subcortical regions involved in emotional regulation and their structural connectivity to the rest of the brain. 

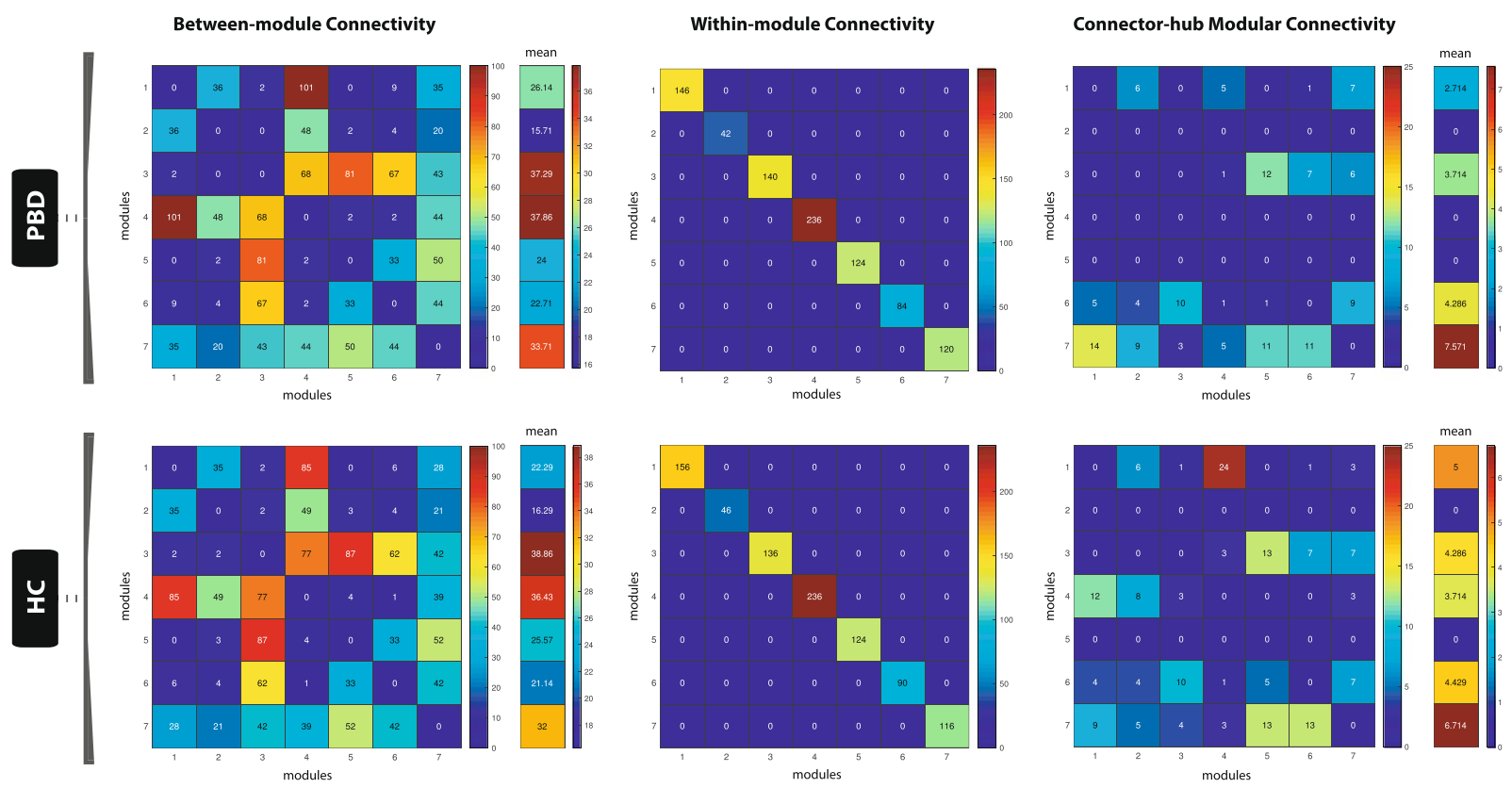

Figure 5. Modular network analysis. The figure shows the patterns of inter-modular (left column), intramodular (middle column) and connector-hub driven inter-modular (right column) connectivity, for patients with PBD with psychosis (top row) and HC (bottom row). Modular connectivity strength is quantified as the total number of connections (degree) of all nodes forming a module. The resulting community structure for the HC population was selected as the reference scheme for group modular connectivity analysis, given its higher group goodness-of-fit (i.e. how well the group estimated community structure fits to each subject's individual community structure). The figure shows that the patterns of SC between modules are changed in PBD. Specifically, that the connectivity of module 4 (right hemisphere; thalamus, insula, basal ganglia, anterior cingulate, precentral gyrus and regions covering most of the frontal cortex) is increased to module 1 (right hemisphere; amygdala, hippocampus, parahippocampus, fusiform gyrus, and other regions of the occipital and temporal cortices) and decreased to module 3 (left hemisphere; thalamus, basal ganglia, anterior cingulate and a large extension of the frontal cortex), whereas module 1 has overall increased inter-modular connectivity but decreased connector-hub driven inter-modular connectivity. The latter suggests an increased degree of sparsity in inter-modular linkage for module 1 (i.e. more connections, though a less prominent role of its connector-hubs in mediating inter-modular connectivity). Despite the absence of connector-hubs in module 4 in PBD, its mean inter-modular connectivity strength is the second largest across modules and similar to HC (with two connector-hubs). This suggests that more nodes (with no 'hub' or 'connector' profile) are mediating communication between module 4 and the remaining modules in PBD, thus indicating decreased selectivity and efficiency in processing communication between a network including the left hemisphere limbic, insular and thalamic areas, and the rest of the brain.

Furthermore, our structural connectivity findings can be thought of in terms of providing the supporting connectome for the previously observed functional connectivity changes in PBD. The structural changes in PBD are likely to lead to changes in functional brain activity. Thus far few studies investigating resting state functional connectivity in $\mathrm{BD}$ have been published, but changes in the functional connectivity of the orbitofrontal cortex ${ }^{2,51,57}$, amygdala $^{58}$ and temporal lobe $e^{51,59}$ have been reported. One particular study analysed resting state networks in $\mathrm{BD}$ during the manic state and showed significant changes in resting state connectivity, mainly involving regions associated with the fronto-temporal, ventral-affective and dorsal-cognitive circuits ${ }^{60}$. Recent evidence has linked $\mathrm{BD}$ to a decreased structural connectivity in right hemisphere in a sub-network involving connections between fronto-temporal and temporal areas ${ }^{23,24}$. Additionally, self-report measure of bipolar risk (hypo/mania proneness) has been linked to increased connectivity between the nucleus accumbens (part of the Caudate in the AAL parcellation), amygdala and $\mathrm{mOFC}^{46}$. These observations are consistent with the present structural connectivity findings (e.g. temporal pole to amygdala, amygdala to caudate and parahippocampal gyrus, superior frontal gyrus and pallidum, between orbitofrontal cortices, caudate and pallidum to orbitofrontal cortices). Taken together, our findings strengthen the hypothesis that functional network dysregulation supported by abnormal structural connectivity, is a prominent driver of pathophysiology in PBD with psychosis.

The results from our graph theoretical analysis of the PBD connectome also revealed significant changes on the nodal efficiency measure with a loss of five hubs (right orbitofrontal cortex, left cuneus, bilateral temporal gyrus and left occipital cortex) in the patient group relative to HC. These brain regions have been demonstrated to be involved in emotional processing and regulation. As such, these changes are consistent with the previously reported abnormal prefrontal top-down dysregulation in $\mathrm{BP}^{4,61}$. 

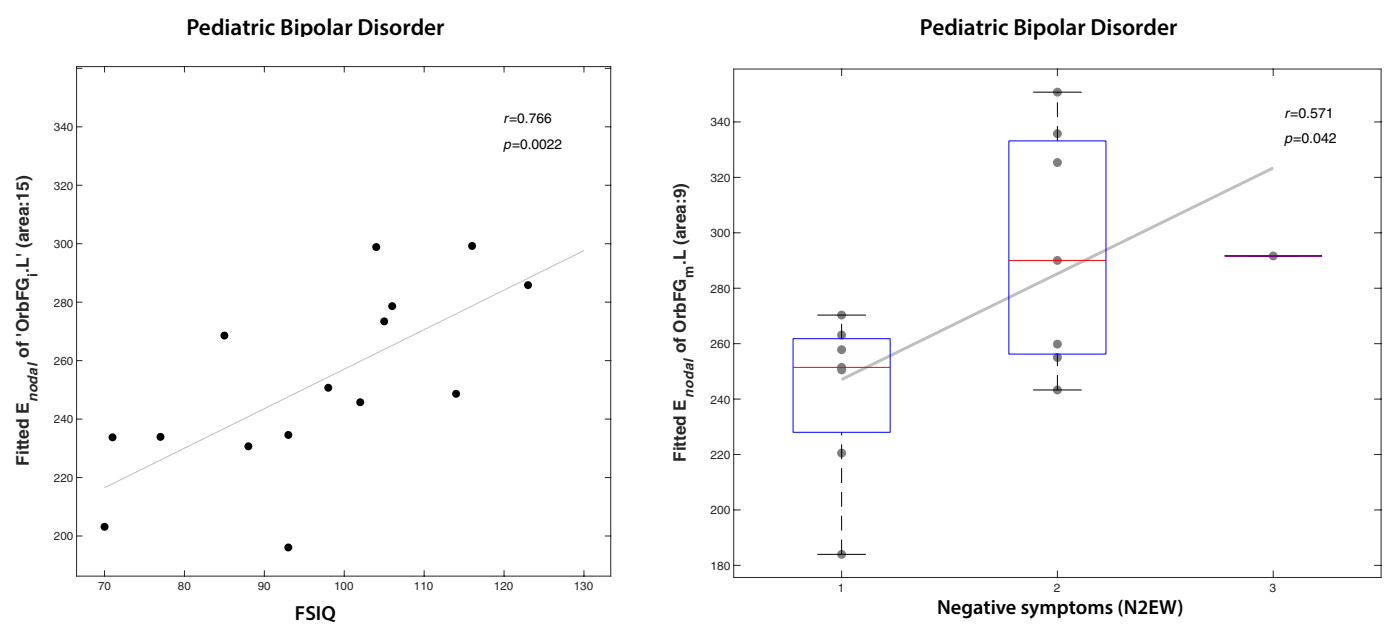

Figure 6. Nodal efficiency of the orbitofrontal cortex in a whole-brain structural connectivity network correlates with emotional withdrawal and IQ in patients with pediatric bipolar disorder (PBD) with psychosis. Left: A positive correlation is found between the nodal efficiency of left inferior orbitofrontal cortex and FSIQ. Right: A positive correlation is also found between the nodal efficiency of the middle orbitofrontal cortex and emotional withdrawal in the PBD group assessed with the PANSS (see Supplementary Table 3 for full details).

We further investigated the whole-brain optimal community structure for both groups. The networks were decomposed into seven modules and we found that the two groups showed differences in modular arrangement, as well as in the fingerprints of inter-modular connectivity. In PDB with psychosis, a large community of brain regions covering most of the right frontal lobe (including the thalamus, insula, basal ganglia, anterior cingulate cortex, amongst other regions), showed increased intra-hemispheric connectivity to an occipito-temporal module (comprising regions such as the amygdala, para/hippocampus, and fusiform gyrus), and decreased inter-hemispheric connectivity to a large module in the left frontal lobe. Additionally, our results suggest that $\mathrm{PBD}$ with psychosis is associated to a decreased degree of centrality in inter-modular connectivity, given a weaker role or inexistence of connector-hubs in mediating inter-modular connectivity, i.e. despite the total number of connections linking modules is similar in PBD, these are mostly driven by a higher number of nodes with a less central role - non-hubs. This effect is found in two right-hemisphere modules - frontal and occipito-temporal, and may be indicative of decreased efficiency in integrating information between these modules and the rest of the brain. Furthermore, significant group differences in participation coefficient were found in a left occipito-temporal module.

The potential functional relevance of these modularity changes can notably be further characterised by investigating the rich club organization. Interestingly, at peak amplitude $\mathrm{k}=35$ for the $\mathrm{HC}$, we found the degree of rich club organisation in the patient group to be lower than in HC. This considerable reduction in rich club organisation suggests lower connectivity between topologically central hub regions of the brain, compared to the healthy participants. To further characterize these changes, we analysed the networks of structural inter-modular connectivity that are driven by 'rich-connector-hubs'; that is, rich-club members that also qualified as connector hubs with high betweeness-centrality and high participation coefficient. Despite the considerable level of overlap in the rich club membership between groups, the integrative nature (participation in inter-modular connectivity) of rich-club members revealed a clear difference in the spatial distribution and number of involved modules, which is suggestive of a disruptive neural network integration capability in the patient group. Specifically, a single right hemisphere module (posterior) was comprised in the rich-connector-hub network, which may reflect a tendency toward weaker global network integration in PBD patients with psychosis.

We also demonstrated that the nodal efficiency of two subdivisions of the orbitofrontal cortex, revealed a subtle association with IQ scores and affective psychotic symptoms in PBD patients. Although the significance of these results did not survive correction for multiple comparisons, it is consistent with prior studies involving the orbitofrontal cortex in emotional processing and deserves further investigations ${ }^{62}$. Furthermore, structural connectivity changes may result not only in the emotional dysfunction seen in PBD but also in significant cognitive deficits as revealed in a recent meta-analysis of verbal learning and memory, processing speed, and executive dysfunction ${ }^{63}$. Our findings support the recently proposed model of BD as "psychosis of interoception", in which the affective dysregulation of BD is a result of dynamic instabilities in introspective circuits, comprising prefrontal and limbic areas, with direct impact on the fear circuitry and cognitive control regions ${ }^{64}$.

An inherent limitation of this study regards the small sample size used. Overcoming the challenges of collecting full high-quality neuroimaging datasets (using state-of-the-art MR sequences) of large and phenotypically homogeneous cohorts of PBD with psychosis, will provide the levels of specificity, sensitivity and statistical power necessary to replicate/validate the significance of our findings and elucidate the brain-behaviour trends presented here. Given the reported trend of increased head-motion in the patient group, future studies will benefit from implementing more efficient strategies to contain head motion inside the MR scanner. 
In summary, our results show that there are significant changes in structural connectivity between patients with early-onset bipolar disorder with psychosis compared to healthy matched participants. These changes are primarily manifested in reduced connectivity between inferior, frontal and temporal cortical areas and to regions linked to emotion, memory and executive function, such as amygdala, hippocampus and the basal ganglia. Taken together, these findings suggest that PBD with psychosis is characterised by a dysfunctional prefrontal regulatory mechanism in line with the hypothesis of prefrontal/fronto-limbic top-down dysregulation in $\mathrm{BD}^{4,61,64}$. Moreover, differences in the structural network's topological organisation suggest a significant reduction of overall integrative processing capability, which is associated with the patients' neurocognitive faculties and symptoms. These changes, seen here in PBD with psychosis need to be further explored in a sample with PBD without psychosis. Collective findings may potentially explain the deficits in emotion processing and regulation found in PBD more generally, and ultimately lead to the development of more targeted treatments.

\section{Data Availability}

The datasets generated during and/or analysed during the current study are available from the corresponding author on reasonable request.

\section{References}

1. Mahon, K., Burdick, K. E. \& Szeszko, P. R. A Role for White Matter Abnormalities in the Pathophysiology of Bipolar Disorder. Neurosci. Biobehav. Rev. 34, 533-554 (2010).

2. James, A. et al. Structural brain and neuropsychometric changes associated with pediatric bipolar disorder with psychosis: Imaging pediatric bipolar disorder. Bipolar Disord. 13, 16-27 (2011).

3. Strakowski, S. M. et al. Functional Magnetic Resonance Imaging Brain Activation in Bipolar Mania: Evidence for Disruption of the Ventrolateral Prefrontal-Amygdala Emotional Pathway. Biol. Psychiatry 69, 381-388 (2011).

4. Phillips, M. L. \& Swartz, H. A. A critical appraisal of neuroimaging studies of bipolar disorder: toward a new conceptualization of underlying neural circuitry and roadmap for future research. Am. J. Psychiatry 171, 829-843 (2014).

5. Nusslock, R. et al. Waiting to win: elevated striatal and orbitofrontal cortical activity during reward anticipation in euthymic bipolar disorder adults. Bipolar Disord. 14 (2012).

6. Linke, J. et al. Increased Medial Orbitofrontal and Amygdala Activation: Evidence for a Systems-Level Endophenotype of Bipolar I Disorder. Am. J. Psychiatry 169, 316-325 (2012).

7. Wessa, M. et al. Fronto-Striatal Overactivation in Euthymic Bipolar Patients During an Emotional Go/NoGo Task. Am. J. Psychiatry 164, 638-646 (2007).

8. Pavuluri, M. N., Passarotti, A. M., Harral, E. M. \& Sweeney, J. A. An fMRI Study of the Neural Correlates of Incidental Versus Directed Emotion Processing in Pediatric Bipolar Disorder. J. Am. Acad. Child Adolesc. Psychiatry 48, 308-319 (2009).

9. Altshuler, L. et al. Regional brain changes in bipolar I depression: a functional magnetic resonance imaging study. Bipolar Disord. 10, 708-717 (2008)

10. Foland, L. C. et al. Evidence for deficient modulation of amygdala response by prefrontal cortex in bipolar mania. Psychiatry Res. Neuroimaging 162, 27-37 (2008).

11. Bora, E., Fornito, A., Yücel, M. \& Pantelis, C. Voxelwise Meta-Analysis of Gray Matter Abnormalities in Bipolar Disorder. Biol. Psychiatry 67, 1097-1105 (2010).

12. Ellison-Wright, I. \& Bullmore, E. Anatomy of bipolar disorder and schizophrenia: A meta-analysis. Schizophr. Res. 117, 1-12 (2010).

13. Selvaraj, S. et al. Grey matter differences in bipolar disorder: a meta-analysis of voxel-based morphometry studies. Bipolar Disord. 14, 135-145 (2012).

14. Wise, T. et al. Voxel-Based Meta-Analytical Evidence of Structural Disconnectivity in Major Depression and Bipolar Disorder. Biol. Psychiatry 79, 293-302 (2016).

15. Abé, C. et al. Cortical thickness, volume and surface area in patients with bipolar disorder types I and II. J. Psychiatry Neurosci. JPN 41, 240-250 (2016).

16. Collin, G. et al. Brain network analysis reveals affected connectome structure in bipolar I disorder: The Structural Connectome in Bipolar Disorder. Hum. Brain Mapp. 37, 122-134 (2016).

17. Dong, D. et al. Shared abnormality of white matter integrity in schizophrenia and bipolar disorder: A comparative voxel-based metaanalysis. Schizophr. Res. 185, 41-50 (2017).

18. Adler, C. M. et al. Evidence of White Matter Pathology in Bipolar Disorder Adolescents Experiencing Their First Episode of Mania: A Diffusion Tensor Imaging Study. Am. J. Psychiatry 163, 322-324 (2006).

19. Barnea-Goraly, N., Chang, K. D., Karchemskiy, A., Howe, M. E. \& Reiss, A. L. Limbic and Corpus Callosum Aberrations in Adolescents with Bipolar Disorder: A Tract-Based Spatial Statistics Analysis. Biol. Psychiatry 66, 238-244 (2009).

20. Kafantaris, V. et al. Lower Orbital Frontal White Matter Integrity in Adolescents With Bipolar I Disorder. J. Am. Acad. Child Adolesc. Psychiatry 48, 79-86 (2009).

21. Goldstein, B. I. \& Birmaher, B. Prevalence, clinical presentation and differential diagnosis of pediatric bipolar disorder. Isr. J. Psychiatry Relat. Sci. 49, 3-14 (2012).

22. Lewinsohn, P. M., Klein, D. N. \& Seeley, J. R. Bipolar disorders in a community sample of older adolescents: prevalence, phenomenology, comorbidity, and course. J. Am. Acad. Child Adolesc. Psychiatry 34, 454-463 (1995).

23. Roberts, G. et al. Structural dysconnectivity of key cognitive and emotional hubs in young people at high genetic risk for bipolar disorder. Mol. Psychiatry 23, 413-421 (2018).

24. O'Donoghue, S., Holleran, L., Cannon, D. M. \& McDonald, C. Anatomical dysconnectivity in bipolar disorder compared with schizophrenia: A selective review of structural network analyses using diffusion MRI. J. Affect. Disord. 209, 217-228 (2017).

25. Bullmore, E. \& Sporns, O. Complex brain networks: graph theoretical analysis of structural and functional systems. Nat. Rev. Neurosci. 10, 186-198 (2009).

26. Bassett, D. S. \& Sporns, O. Network neuroscience. Nat. Neurosci. 20, 353-364 (2017).

27. Deco, G. \& Kringelbach, M. L. Great Expectations: Using Whole-Brain Computational Connectomics for Understanding Neuropsychiatric Disorders. Neuron 84, 892-905 (2014).

28. Collin, G., Scholtens, L. H., Kahn, R. S., Hillegers, M. H. J. \& Heuvel, M. Pvanden Affected Anatomical Rich Club and Structural-Functional Coupling in Young Offspring of Schizophrenia and Bipolar Disorder Patients. Biol. Psychiatry 82, 746-755 (2017).

29. Wang, B. et al. The Abnormality of Topological Asymmetry in Hemispheric Brain Anatomical Networks in Bipolar Disorder. Front. Neurosci. 12 (2018).

30. Spielberg, J. M. et al. Resting State Brain Network Disturbances Related to Hypomania and Depression in Medication-Free Bipolar Disorder. Neuropsychopharmacology 41, 3016-3024 (2016). 
31. Tzourio-Mazoyer, N. et al. Automated Anatomical Labeling of Activations in SPM Using a Macroscopic Anatomical Parcellation of the MNI MRI Single-Subject Brain. NeuroImage 15, 273-289 (2002).

32. Jenkinson, M., Bannister, P., Brady, M. \& Smith, S. Improved Optimization for the Robust and Accurate Linear Registration and Motion Correction of Brain Images. NeuroImage 17, 825-841 (2002).

33. Collins, D. L., Neelin, P., Peters, T. M. \& Evans, A. C. Automatic 3D intersubject registration of MR volumetric data in standardized Talairach space. J. Comput. Assist. Tomogr. 18, 192-205 (1994).

34. Behrens, T. E. J. et al. Characterization and propagation of uncertainty in diffusion-weighted MR imaging. Magn. Reson. Med. 50, 1077-1088 (2003).

35. Behrens, T. E. J., Berg, H. J., Jbabdi, S., Rushworth, M. F. S. \& Woolrich, M. W. Probabilistic diffusion tractography with multiple fibre orientations: What can we gain? NeuroImage 34, 144-155 (2007).

36. Zalesky, A. et al. Connectome sensitivity or specificity: which is more important? NeuroImage 142, 407-420 (2016).

37. Roberts, J. A., Perry, A., Roberts, G., Mitchell, P. B. \& Breakspear, M. Consistency-based thresholding of the human connectome. NeuroImage 145, 118-129 (2017).

38. Baum, G. L. et al. The impact of in-scanner head motion on structural connectivity derived from diffusion MRI. NeuroImage 173, 275-286 (2018).

39. Aoki, Y., Cortese, S. \& Castellanos, F. X. Research Review: Diffusion tensor imaging studies of attention-deficit/hyperactivity disorder: meta-analyses and reflections on head motion. J. Child Psychol. Psychiatry 59, 193-202 (2018).

40. Rubinov, M. \& Sporns, O. Complex network measures of brain connectivity: Uses and interpretations. NeuroImage 52, 1059-1069 (2010).

41. Blondel, V. D., Guillaume, J.-L., Lambiotte, R. \& Lefebvre, E. Fast unfolding of communities in large networks. J. Stat. Mech. Theory Exp. 2008, P10008 (2008)

42. Achard, S. \& Bullmore, E. Efficiency and Cost of Economical Brain Functional Networks. PLOS Comput. Biol. 3, e17 (2007).

43. Lo, C.-Y. et al. Diffusion Tensor Tractography Reveals Abnormal Topological Organization in Structural Cortical Networks in Alzheimer's Disease. J. Neurosci. 30, 16876-16885 (2010)

44. Zalesky, A., Fornito, A. \& Bullmore, E. T. Network-based statistic: Identifying differences in brain networks. NeuroImage 53, 1197-1207 (2010).

45. Nelson, B. G., Bassett, D. S., Camchong, J., Bullmore, E. T. \& Lim, K. O. Comparison of large-scale human brain functional and anatomical networks in schizophrenia. NeuroImage Clin. 15, 439-448 (2017).

46. Damme, K. S., Young, C. B. \& Nusslock, R. Elevated nucleus accumbens structural connectivity associated with proneness to hypomania: a reward hypersensitivity perspective. Soc. Cogn. Affect. Neurosci. 12, 928-936 (2017).

47. Brambilla, P., Bellani, M., Yeh, P.-H. \& Soares, J. C. Myelination in bipolar patients and the effects of mood stabilizers on brain anatomy. Curr. Pharm. Des. 15, 2632-2636 (2009).

48. Heng, S., Song, A. W. \& Sim, K. White matter abnormalities in bipolar disorder: insights from diffusion tensor imaging studies. J. Neural Transm. 117, 639-654 (2010).

49. Borgwardt, S. \& Fusar-Poli, P. White matter pathology-an endophenotype for bipolar disorder? BMC Psychiatry 12, 138 (2012).

50. Lim, C. S. et al. Longitudinal neuroimaging and neuropsychological changes in bipolar disorder patients: Review of the evidence. Neurosci. Biobehav. Rev. 37, 418-435 (2013).

51. Wilke, M., Kowatch, R. A., DelBello, M. P., Mills, N. P. \& Holland, S. K. Voxel-based morphometry in adolescents with bipolar disorder: first results. Psychiatry Res. 131, 57-69 (2004)

52. Gogtay, N. et al. Dynamic mapping of cortical development before and after the onset of pediatric bipolar illness. J. Child Psychol. Psychiatry 48, 852-862 (2007).

53. Kaur, S. et al. Cingulate Cortex Anatomical Abnormalities in Children and Adolescents With Bipolar Disorder. Am. J. Psychiatry 162, 1637-1643(2005).

54. Dickstein, D. P. et al. Frontotemporal alterations in pediatric bipolar disorder: results of a voxel-based morphometry study. Arch. Gen. Psychiatry 62, 734-741 (2005).

55. Najt, P. et al. Anterior Cortical Development During Adolescence in Bipolar Disorder. Biol. Psychiatry 79, 303-310 (2016).

56. Frazier, J. A. et al. Structural Brain Magnetic Resonance Imaging of Limbic and Thalamic Volumes in Pediatric Bipolar Disorder. Am. J. Psychiatry 162, 1256-1265 (2005)

57. Dickstein, D. P. et al. Comorbid anxiety in phenotypes of pediatric bipolar disorder. J. Child Adolesc. Psychopharmacol. 15, 534-548 (2005)

58. Pfeifer, J. C., Welge, J., Strakowski, S. M., Adler, C. M. \& DelBello, M. P. Meta-analysis of amygdala volumes in children and adolescents with bipolar disorder. J. Am. Acad. Child Adolesc. Psychiatry 47, 1289-1298 (2008).

59. Frazier, J. A. et al. Cortical gray matter differences identified by structural magnetic resonance imaging in pediatric bipolar disorder. Bipolar Disord. 7, 555-569 (2005).

60. Xiao, Q. et al. Altered Regional Homogeneity in Pediatric Bipolar Disorder during Manic State: A Resting-State fMRI Study. PLoS ONE 8, e57978 (2013).

61. Strakowski, S. M. et al. The functional neuroanatomy of bipolar disorder: a consensus model. Bipolar Disord. 14 (2012).

62. Kringelbach, M. L. The human orbitofrontal cortex: linking reward to hedonic experience. Nat. Rev. Neurosci. 6, 691-702 (2005).

63. Nieto, R. G. \& Castellanos, F. X. A meta-analysis of neuropsychological functioning in patients with early onset schizophrenia and pediatric bipolar disorder. J. Clin. Child Adolesc. Psychol. Off. J. Soc. Clin. Child Adolesc. Psychol. Am. Psychol. Assoc. Div. 53 40, 266-280 (2011).

64. Perry, A., Roberts, G., Mitchell, P. B. \& Breakspear, M. Correction: Connectomics of bipolar disorder: a critical review, and evidence for dynamic instabilities within interoceptive networks. Mol. Psychiatry 1, https://doi.org/10.1038/s41380-018-0327-7 (2019).

\section{Acknowledgements}

The authors gratefully thank Mikkel Petersen for his help in the DTI analysis and Angus Stevner for his inputs on group consistency testing. M.L.K. was supported by the ERC Consolidator Grant: CAREGIVING (No. 615539), TrygFonden Charitable Foundation and by Center for Music in the Brain, funded by the Danish National Research Foundation (DNRF117). J.C. was supported by the Portuguese Foundation for Science and Technology CEECIND/03325/2017, Portugal). G.D. was supported by the Spanish Research Project SAF2010-16085 and the FP7-ICT BrainScales. The clinical and neuroimaging data used in this study was supported by the Medical Research Council (M.R.C. G0500092) and the Oxford Hospital Services Research Committee (OHSCR).

\section{Author Contributions}

H.M.F. designed and implemented the methodological framework used in this study. H.M.F., C.G. and R.J.v.H. processed the data. H.M.F., M.L.K., J.C., P.P. and L.D.L. wrote the manuscript. A.J. performed the clinical assessment of all patients and healthy participants and provided the financial support for the neuroimaging data collection. A.J., G.D., P.C.W. and A.M. acted in an advisory and editorial capacity. 


\section{Additional Information}

Supplementary information accompanies this paper at https://doi.org/10.1038/s41598-019-50093-4.

Competing Interests: The authors declare no competing interests.

Publisher's note Springer Nature remains neutral with regard to jurisdictional claims in published maps and institutional affiliations.

(c) (i) Open Access This article is licensed under a Creative Commons Attribution 4.0 International License, which permits use, sharing, adaptation, distribution and reproduction in any medium or format, as long as you give appropriate credit to the original author(s) and the source, provide a link to the Creative Commons license, and indicate if changes were made. The images or other third party material in this article are included in the article's Creative Commons license, unless indicated otherwise in a credit line to the material. If material is not included in the article's Creative Commons license and your intended use is not permitted by statutory regulation or exceeds the permitted use, you will need to obtain permission directly from the copyright holder. To view a copy of this license, visit http://creativecommons.org/licenses/by/4.0/.

(C) The Author(s) 2019 